\title{
Topological susceptibility through the deconfining phase transition*
}

\author{
B. Allés ${ }^{\mathrm{a}}$ M. D'Elia ${ }^{\mathrm{a} \dagger}$ and A. Di Giacomo ${ }^{\mathrm{a}}$

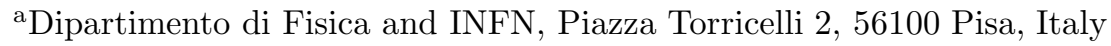

We present a measurement of the topological susceptibility in $S U(3)$ Yang-Mills theory through the deconfinement phase transition. An improved operator is used for the topological charge density. A drop by an order of magnitude is observed from the confined to the deconfined phase.

\section{INTRODUCTION}

The topological susceptibility is defined in continuum QCD as

$\chi \equiv \int d^{4} x\langle 0|T(Q(x) Q(0))| 0\rangle$,

where

$Q(x)=\frac{g^{2}}{64 \pi^{2}} \epsilon^{\mu \nu \rho \sigma} F_{\mu \nu}^{a}(x) F_{\rho \sigma}^{a}(x)$.

$Q(x)$ is the topological charge density and is proportional to the anomaly of the $U_{A}(1)$ current [1]

$\partial_{\mu} j_{5}^{\mu}(x)=2 N_{f} Q(x)$.

The value of $\chi$ for quenched QCD can be related to the masses of the pseudoscalar mesons, by use of $\frac{1}{N_{c}}$ expansion [2, 3

$\frac{2 N_{f}}{f_{\pi}^{2}} \chi=m_{\eta}^{2}+m_{\eta^{\prime}}^{2}-2 m_{K}^{2}$.

$\chi$ has been determined on the lattice [A] and is consistent with the prediction of eq. (4). This solves the so-called $U_{A}(1)$ problem [5].

It is interesting to determine the behaviour of $\chi$ at $T \neq 0$, in particular across the deconfining phase transition, which in quenched QCD takes place at $T_{c} \sim 260 \mathrm{MeV}$ [6]. A general expectation is that the topological susceptibility should drop at $T_{c}$ [7], since Debye screening inhibits tunneling between states of different chirality and damps the density of instantons.

Previous attempts to study $\chi$ through $T_{c}$ by the field theoretical method were plagued with

\footnotetext{
*Pisa preprint, IFUP-TH 43/96

${ }^{\dagger}$ Speaker at the conference.
}

large statistical uncertainties above $T_{c}$ [8]. Also the attempts to use the cooling technique encountered difficulties at finite $T$ [8,9]. The idea of the cooling method [9] is to measure the topological charge on configurations cooled by locally minimizing the action. At $T=0$ and below $T_{c}$ the method works well and instantons show up as plateaux of the topological charge versus cooling. Around $T_{c}$ instantons become unstable, the plateaux disappear and the method becomes ambiguous [8].

In the present paper we drastically reduce the statistical fluctuations of the field theoretical method determination, by using an improved topological charge operator as proposed in ref. 10].

We redetermine $\chi$ at $T=0$ and study its behaviour through $T_{c}$. The new determination of $\chi$ at $T=0$ is consistent with previous determinations 11 13. At finite $T$ we obtain a good determination of $\chi$. Our main result is that $\chi$ drops at $T_{c}$ by more than one order of magnitude.

\section{THE METHOD}

The field theoretical method is a straightforward application of the basic rules and concepts of quantum field theory.

At first a lattice discretization $Q_{L}(x)$ of the continuum topological charge density operator $Q(x)$ is defined. $Q_{L}(x)$ is not unique: infinitely many operators can be defined on the lattice which have the same continuum limit.

In going to the continuum limit a proper renormalization must be performed, like in any other regularization scheme. In quenched QCD $Q_{L}$ 
renormalizes multiplicatively [14]. In formulae

$Q_{L}=Z(\beta) Q a^{4}+\mathcal{O}\left(a^{6}\right)$.

As usual, $\beta \equiv 6 / g_{0}^{2}$. The topological susceptibility can be defined on the lattice as

$\chi_{L} \equiv\left\langle\sum_{x} Q_{L}(x) Q_{L}(0)\right\rangle$.

The standard rules of renormalization then give

$\chi_{L}=Z(\beta)^{2} a^{4} \chi+M(\beta)+\mathcal{O}\left(a^{6}\right)$,

where $M(\beta)$ is an additive renormalization containing mixings of $\chi_{L}$ to other operators with the same quantum numbers and lower or equal dimensions 15.

$Z$ and $M$ can be computed non-perturbatively on the lattice by the heating method [12,16,17. Renormalizations are induced by quantum fluctuations at the scale of the UV cutoff (lattice spacing scale), so they can be estimated by measuring topological quantities on an ensemble of configurations where the topological content is known and short-range fluctuations are thermalized. In order to do this one can start with a classical configuration on the lattice and perform few local updating steps on it until the short-range fluctuations are thermalized: by repeating this procedure several times an ensemble of trajectories can be constructed, all starting from the same initial configuration. Starting from a large instanton and measuring $Q_{L}$ on the ensemble, a plateau at the value of $Z(\beta)$ is reached with respect to the number of heating step performed. Similarly starting from the flat configuration and measuring $\chi_{L}$ a plateau at the value of $M(\beta)$ is reached. To be sure that the initial topology is not changed by the heating procedure, each configuration of the sample is checked during heating by performing a few cooling steps to detect its topological charge on a copy of it. If a change occurred the configuration is discarded [18].

Once $M$ and $Z$ are known, $\chi$ can be extracted by a subtraction procedure following eq. (7). Clearly $Z$ and $M$ depend on the operator used for $Q_{L}$. A standard definition for $Q_{L}$ is 19

$Q_{L}(x)=\frac{-1}{2^{9} \pi^{2}} \sum_{\mu \nu \rho \sigma= \pm 1}^{ \pm 4} \tilde{\epsilon}_{\mu \nu \rho \sigma} \operatorname{Tr}\left(\Pi_{\mu \nu} \Pi_{\rho \sigma}\right)$.
Here $\tilde{\epsilon}_{\mu \nu \rho \sigma}$ is the standard Levi-Civita tensor for positive directions while for negative ones the relation $\tilde{\epsilon}_{\mu \nu \rho \sigma}=-\tilde{\epsilon}_{-\mu \nu \rho \sigma}$ holds. $\Pi_{\mu \nu}$ is the plaquette in the $\mu-\nu$ plane at point $x$. With this definition $Z \simeq 0.18$ and the mixing $M$ is most of the measured signal in the scaling region [11], so that a large error derives from the subtraction procedure performed to extract $\chi$.

In order to obtain a more precise determination of $\chi$, a new definition of $Q_{L}$ can be used for which $M$ is strongly reduced and $Z$ is closer to its continuum value $Z=1$.

In this work we use the sequence of improved operators $Q_{L}^{(i)}$ defined in ref. [10]: the starting operator of the sequence is the standard definition of eq. (8) and a smearing procedure is performed at each step of the sequence. We stop at the second smearing, where a considerable improvement is already achieved. For a full account of our results see ref. [20].

\section{RESULTS}

We measured the topological susceptibility at zero temperature on a symmetric lattice $16^{4}$ and at finite temperature on a lattice $32^{3} \times$ 8. The simulations were performed on a APE QUADRICS machine.

In figure 1 we plot the value of $(\chi)^{(1 / 4)}$ at zero temperature versus $\beta$ for the three definitions $\chi_{L}^{(i)}$ $(i=0,1,2)$ of the lattice topological susceptibility. There is good scaling: $(\chi)^{(1 / 4)}$ is independent of $\beta$, as it should. There is also an excellent agreement between the three determinations. To fix the value of $(\chi)^{(1 / 4)}$ in physical units we use for $a(\beta)$ the two loop formula and for $\Lambda_{L}$ the determination of ref. 21] $\Lambda_{L}=4.56(11) \mathrm{MeV}$. The horizontal line is the linear fit to the 2 -smear data. It gives $(\chi)^{(1 / 4)}=175(5) \mathrm{MeV}$ and is consistent, within errors, with that of ref. 111 13. The error includes the uncertainty in $\Lambda_{L}$.

In figure 2 the topological susceptibility $\chi \equiv$ $\left(\chi_{L}^{(i)}-M^{(i)}\right) /\left(Z^{(i) 2} a^{4}\right)$ at the transition point is shown for the 1 and 2 -smeared operator. $\chi$ drops by one order of magnitude from the confined to the deconfined phase. The results obtained with the two operators are compatible as they should. The data for the 0-smeared operator have very 


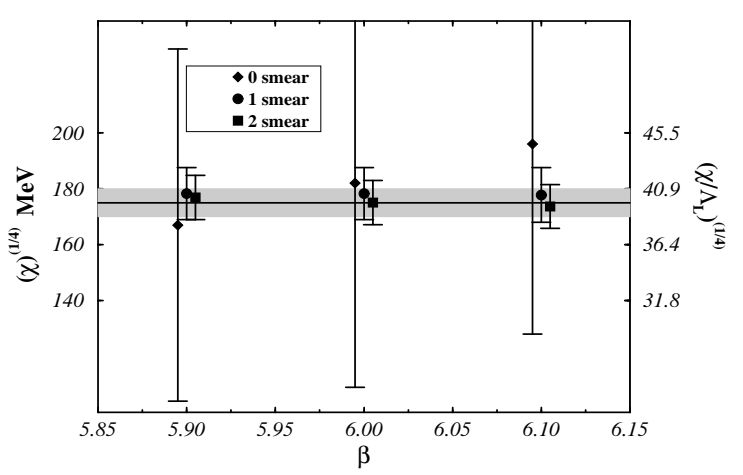

Figure 1. $\chi$ at $T=0$. The straight line is the result of the linear fit of the 2 -smeared data. The improvement from $Q_{L}^{(0)}$ to $Q_{L}^{(2)}$ is clearly visible.

large error bars and are not shown in the figure. The data have been plotted versus $T / T_{c}$ where $T_{c}$ is the deconfining temperature coresponding to $\beta_{c}\left(N_{\tau}=8\right)=6.0609(9)$ [6]. To determine $T / T_{c}$ we only need the ratio $a\left(\beta_{c}\right) / a(\beta)$ and for that the two-loop expression is certainly a good approximation within the small interval of $\beta$ used, where $\Lambda_{L}$ can be considered as a constant.

The solid line in figure 5 corresponds to the value of $\chi$ at zero-temperature and is consistent with the data below $T_{c}$ indicating that $\chi$ is practically $T$-independent in the confined phase.

We thank Graham Boyd and Enrico Meggiolaro for useful discussions. A.D.G. acknowledges an interesting discussion with Heinrich Leutwyler.

\section{REFERENCES}

1. G. t'Hooft, Phys. Rev. Lett. 37 (1976) 8.

2. E. Witten, Nucl. Phys. B156 (1979) 269.

3. G. Veneziano, Nucl. Phys. B159 (1979) 213.

4. For a review see e.g. A. Di Giacomo, Nucl. Phys. (Proc. Suppl.) B23 (1981) 191.

5. S. Weinberg, Phys. Rev. D11 (1975) 3583.

6. G. Boyd, J. Engels, F. Karsch, E. Laermann, C. Legeland, M. Lütgemeier and B. Petersson, hep-lat 9602007.

7. R. D. Pisarski, L.G.Yaffe, Phys. Lett. B97 (1980) 110.

8. A. Di Giacomo, E. Meggiolaro,

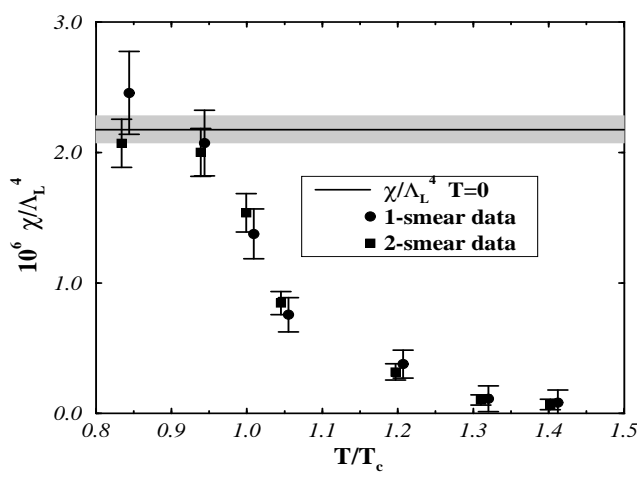

Figure 2. $\chi / \Lambda_{L}^{4}$ versus $T / T_{c}$ across the deconfining phase transition. The horizontal band is the determination at $T=0$ of figure 1 .

H. Panagopoulos, Phys. Lett. B277 (1992) 491.

9. M. Teper, Phys. Lett. B171 (1986) 81, 86.

10. C. Christou, A. Di Giacomo, H. Panagopoulos and E. Vicari, Phys. Rev. D53 (1996) 2619.

11. M. Campostrini, A. Di Giacomo, Y. Gündüç, M. P. Lombardo, H. Panagopoulos, R. Tripiccione, Phys. Lett. B252 (1990) 436.

12. B. Allés, M. Campostrini, A. Di Giacomo, Y. Gündüç and E. Vicari, Nucl. Phys. (Proc. Suppl.) B34 (1994) 504.

13. M. Teper, Phys. Lett. B202 (1988) 553.

14. M. Campostrini, A. Di Giacomo and H. Panagopoulos, Phys. Lett. B212 (1988) 206.

15. M. Campostrini, A. Di Giacomo, H. Panagopoulos and E. Vicari, Nucl. Phys. B329 (1990) 683.

16. A. Di Giacomo and E. Vicari, Phys. Lett. B275 (1992) 429.

17. B. Allés, M. Campostrini, A. Di Giacomo, Y. Gündüç and E. Vicari, Phys. Rev. D48 (1993) 2284.

18. F. Farchioni and A. Papa, Nucl. Phys. B431 (1994) 686.

19. P. Di Vecchia, K. Fabricius, G.C. Rossi, G. Veneziano, Nucl. Phys. B192 (1981) 392.

20. B. Allés, M. D'Elia, A. Di Giacomo, Pisa preprint, IFUP-TH 26/96 and hep-lat 9605013.

21. G. S. Bali and K. Schilling, Phys. Rev. D47 (1993) 661. 